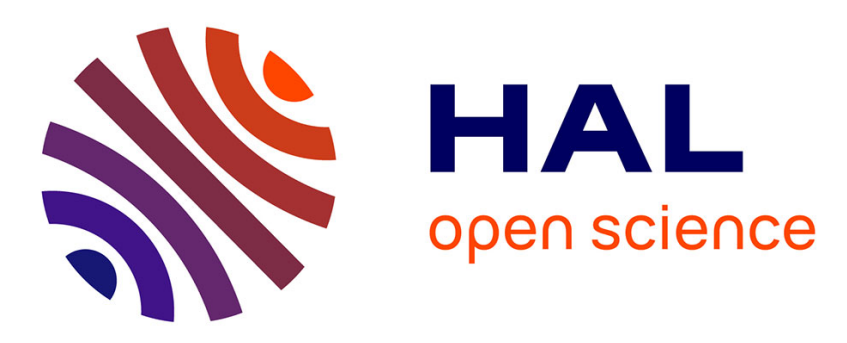

\title{
Electropreconcentration diagrams to optimize molecular enrichment with low counter pressure in a nanofluidic device
}

\author{
Sokhna-mery Ngom, Fatima Flores-galicia, François-damien Delapierre, \\ Antoine Pallandre, Jean Gamby, Isabelle Le Potier, Anne-Marie \\ Haghiri-Gosnet
}

\section{To cite this version:}

Sokhna-mery Ngom, Fatima Flores-galicia, François-damien Delapierre, Antoine Pallandre, Jean Gamby, et al.. Electropreconcentration diagrams to optimize molecular enrichment with low counter pressure in a nanofluidic device. Electrophoresis, 2020, 41 (18-19), pp.1617-1626. 10.1002/elps.202000117 . hal-02992532

\section{HAL Id: hal-02992532 \\ https://hal.science/hal-02992532}

Submitted on 22 Dec 2020

HAL is a multi-disciplinary open access archive for the deposit and dissemination of scientific research documents, whether they are published or not. The documents may come from teaching and research institutions in France or abroad, or from public or private research centers.
L'archive ouverte pluridisciplinaire HAL, est destinée au dépôt et à la diffusion de documents scientifiques de niveau recherche, publiés ou non, émanant des établissements d'enseignement et de recherche français ou étrangers, des laboratoires publics ou privés. 


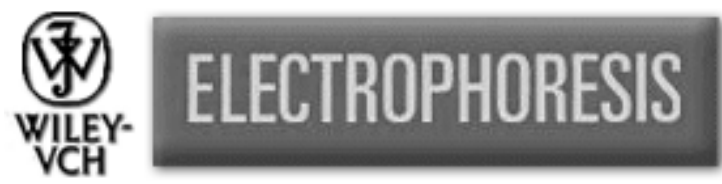

\section{Electropreconcentration diagrams to optimize molecular enrichment with low counter pressure in a nanofluidic device.}

\begin{tabular}{|r|l|}
\hline Journal: & ELECTROPHORESIS \\
\hline Manuscript ID & Draft \\
\hline Wiley - Manuscript type: & Research Paper \\
\hline Author: Submitted by the & $\mathrm{n} / \mathrm{a}$ \\
\hline Complete List of Authors: & $\begin{array}{l}\text { Ngom, Sokhna-Mery; C2N, Microsystems and Nanobiofluidics } \\
\text { Flores-Galicia, Fatima; C2N, Microsystems and Nanobiofluidics } \\
\text { Delapierre, François-Damien; C2N, Microsystems and Nanobiofluidics } \\
\text { Pallandre, Antoine; Laboratoire de chimie physique d'Orsay, Chemistry } \\
\text { Gamby, Jean; C2N, Microsystems and Nanobiofluidics } \\
\text { Le Potier, Isabelle; C2N, Microsystems and Nanobiofluidics } \\
\text { Haghiri-Gosnet, Anne-Marie; C2N, Microsystems and Nanobiofluidics }\end{array}$ \\
\hline Keywords: & Nanofluidics, Electrokinetic transport, Preconcentration diagrams \\
\hline &
\end{tabular}

\section{SCHOLARONE \\ Manuscripts}




\title{
Electropreconcentration diagrams to optimize
}

\section{molecular enrichment with low counter pressure in a}

\author{
nanofluidic device.
}

\author{
Sokhna-Mery Ngom ${ }^{a}$, Fatima Flores-Galicia ${ }^{a}$, François-Damien Delapierre ${ }^{a}$, Antoine \\ Pallandre ${ }^{b \perp}$, Jean Gamby ${ }^{a \perp}$, Isabelle Le Potier ${ }^{a \perp}$ and Anne-Marie Haghiri-Gosnet ${ }^{a *}$. \\ ${ }^{a}$ Centre de Nanosciences et Nanotechnologies C2N, CNRS, Université Paris-Saclay, UMR9001, \\ 10 Boulevard Thomas Gobert, 91120 Palaiseau, France \\ ${ }^{\mathrm{b}}$ Université Paris-Saclay, CNRS, Institut de Chimie Physique, UMR8000, 91405 Orsay, France \\ ${ }^{\perp}$ These authors have equally contributed to the paper.
}

\begin{abstract}
:
Ion-concentration-polarization (ICP) - based focusing electrokinetics nanofluidic devices have been developed in order to simultaneously detect and enrich very diluted analytes on chip. However, stabilization of focal points over long time under the application of the electric field remains as a technical bottleneck. If pressure-assisted preconcentration methods have been proposed to stabilize propagating modes at $1 / D_{u} \ll 1$, these recent protocols remain laborious for
\end{abstract}


optimizing experimental parameters. We report "field/pressure" E/P diagrams for fluorescein where the typical regimes, i.e. propagating focusing, stable focusing and stacking can be observed. The region of stable focusing is shown to vary depending of the nanoslit length $\left(100 \mu \mathrm{m}<\mathrm{L}_{\text {nanoslit }}\right.$ $<500 \mu \mathrm{m})$ and the nature of the background electrolyte (BGE) $(\mathrm{KCl}$ and $\mathrm{NaCl})$. Longer nanoslits $(500 \mu \mathrm{m})$ produce stabilization at low pressure, whereas $\mathrm{NaCl}$ BGE offers a narrower and more fluorescent stable window in the $\mathrm{E} / \mathrm{P}$ diagram compared to $\mathrm{KCl}$. Finally, the ability of such pressure-assisted protocol to concentrate negatively charged proteins has been tested with $10 \mu \mathrm{M}$ ovalbumin in HEPES and the corresponding E/P diagram for ovalbumin confirms the existence of a stable focusing regime at low electric field.

Keywords: Nanofluidics, Electrokinetic transport, Preconcentration diagrams

*Corresponding author: anne-marie.haghiri@c2n.upsaclay.fr

Co-corresponding author: antoine.pallandre@u-psud.fr 


\section{Introduction}

In the field of highly sensitive biochemical analysis, Lab-on-a-Chip (LoC) devices have to face the challenge of maintaining a good level of sensitivity and selectivity, while decreasing the amount of analyte. The analysis of highly diluted biomarkers produced at the very early stages of a disease is always facing the issue of reaching the relevant sensitivity to provide a reliable diagnosis. It is therefore crucial to couple good separation with sensitive and specific detection [1]. Separation methods that consist in controlling the spatial location of the analyte in the fluid over time [2-7] may suffer from a lack of sensitivity since there is no preconcentration. In this context developing new solutions for sample analyte preconcentration in bioanalytical fluidic devices remains a necessity. To address this prevalent issue, many focusing techniques based on electrokinetic phenomena have been proposed, among which ion concentration polarization [820], field-amplified sample stacking (FASS) [21-23], concentration gradient focusing [24-27] and isoelectric focusing [28-30]. All these techniques allow focusing analytes by exploiting the unbalanced ionic transport between anionic and cationic species due to the competition between electroosmotic flow (EOF) and electrophoretic flow (EP).

This unbalanced transport under electric field is exacerbated in a nanofluidic device that integrates channels with at least one nanometric dimension [6]. In such nanofluidic device filled with a background electrolyte (BGE), this exacerbated unbalanced transport produces an enrichment-exclusion effect that is called the ion concentration polarization $(\mathrm{CP})$ phenomenon [910]. This CP effect is mainly governed by the background electrolyte (BGE) concentration and the geometrical constraints imposed by the nanofluidic geometry. At each side of the nanochannel, $\mathrm{CP}$ generates BGE enrichment (cathodic reservoir) and depletion (anodic reservoir) zones with respectively a high and a low conductivity. These conductivity gradients and the corresponding 
gradients in the local electromigration velocity of analytes produce a stacking/focusing phenomenon at the interfaces of enrichment (cathodic reservoir) and depletion (anodic reservoir) shocks. These shocks are net boundaries where the analyte can locate and thus concentrate. Based on a general analytical theory of $\mathrm{CP}[9,11]$, the various theoretically predicted mechanisms for $\mathrm{CP}$ of the BGE are governed by the two key parameters:

$1 /$ an inverse Dukhin number $1 / D_{u}$, which describes the ratio of bulk conductivity to surface conductivity, defined by $\frac{1}{D_{u}}=\frac{G_{b u l k}}{G_{\sigma}}=\left(\frac{v_{1} z_{1}-v_{2} z_{2}}{-2 v_{1} \sigma}\right) F h_{n} c_{0, r}$, where $v_{i}$ and $z_{i}$ are respectively the mobility and the valence number of the $\mathrm{i}^{\text {th }}$ ion $\left(1\right.$ for the ion $\mathrm{Na}^{+}$or $\mathrm{K}^{+}$and 2 for the co-ion $\left.\mathrm{Cl}^{-}\right), F$ is the Faraday number, $h_{n}$ is the nanoslit height, $\sigma$ is the surface charge and $c_{0, r}$ is the concentration of the co-ion in the reservoir.

$2 /$ the velocity of the co-ion (to the wall charge) nondimensionalized by the electroosmotic velocity $v_{2}^{*}=\frac{v_{2} z_{2} F \eta}{\zeta_{n} \varepsilon}$, where $\zeta_{n}$ is the zeta potential value assumed to be uniform along the structure, $\eta$ is the viscosity and $\varepsilon$ is the permittivity.

At high $1 / D_{u}$ values and low $v_{2}^{*}$, values the concentrated analyte will stack at the entrance of the nanochannel in a regime called "CP without propagation" $\left(1 / D_{u} \gg 1\right)[11]$. Such stacking could not be used for real applications of preconcentration since the analyte is confined in the vicinity of the nanochannel. On the opposite, at low inverse Dukhin number $\left(1 / D_{u} \ll 1\right)$, the analyte will stack at the sharp interface of enrichment shock (cathodic reservoir) or focus at the interface of depletion shock (anodic reservoir) in a regime called "propagating CP". We have previously shown based on computations [12] that an anionic analyte, such as fluorescein, diluted at low BGE concentration (down to $10 \mu \mathrm{M}$ ), will concentrate in the cathodic reservoir in an 
electrophoretically dominated regime. Such a very low ionic strength with high surface charge promotes a cathodic concentration profile unstable over time that corresponds to the propagating CP of the BGE (see Fig. 1(a)) [9]. This type of stacking "cathodic counter gradient focusing" (CCGF)[11,16] will be named here "Cathodic Focusing" (CF) to simplify.

a)

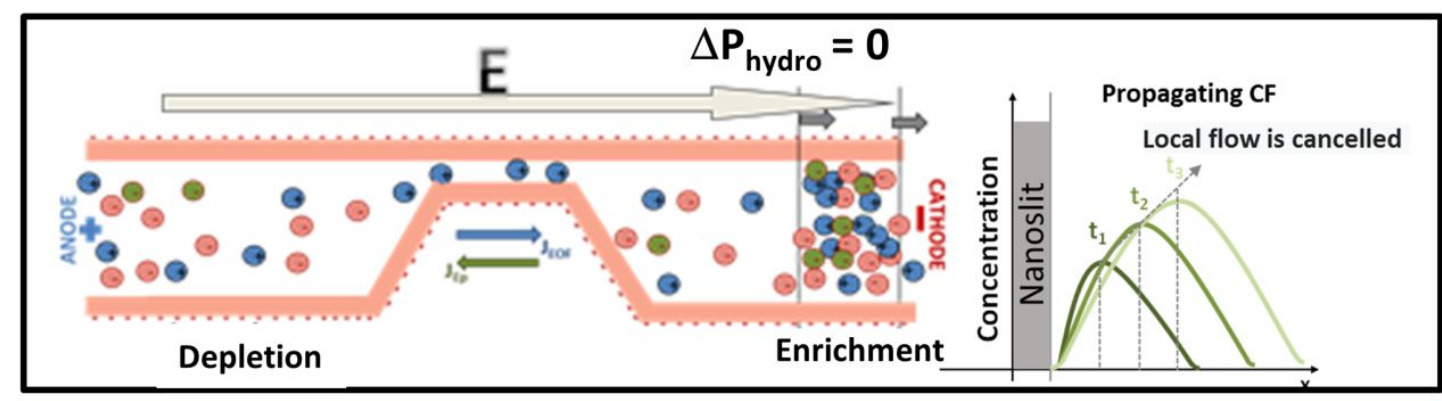

b)

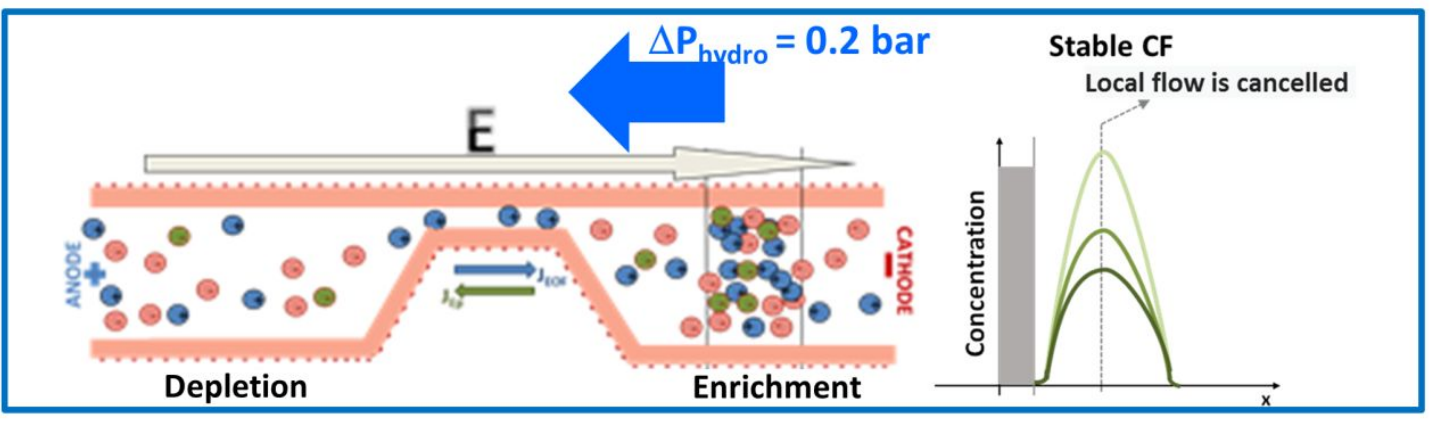

c)

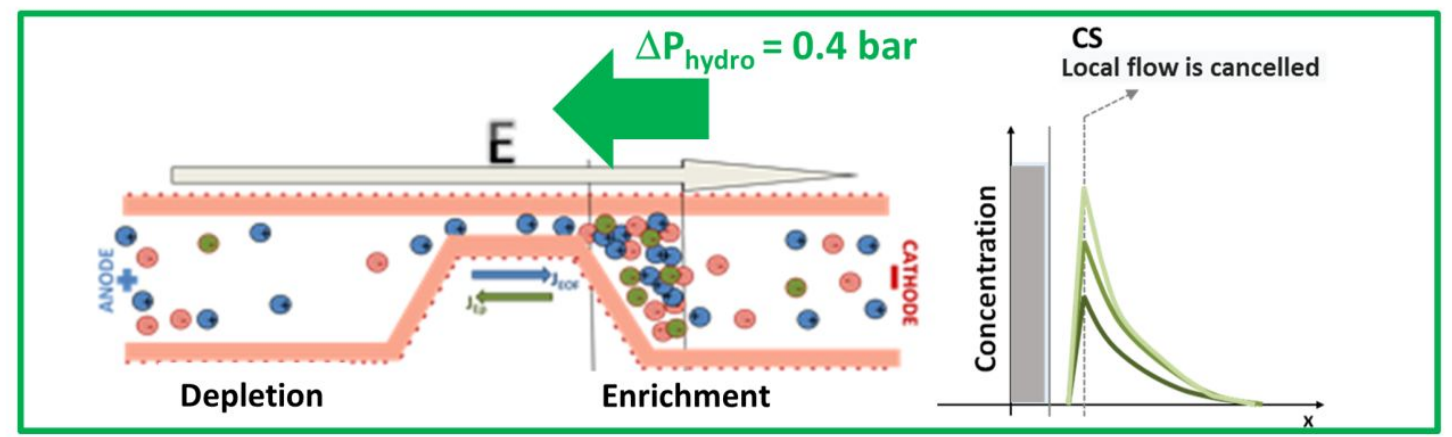

Figure 1. Cross-view of the micro/nano/micro (MNM) device with (a) a typical "propagating CF" profile observed for an anionic analyte stacking and moving at the enrichment shock with $v_{2}^{*}<1$ for conventional electropreconcentration (without any counter-pressure $\Delta P_{\text {hydro }}=0$ ), (b) stabilization of the concentration frontline under the application of a low cathodic counter-pressure $\Delta P_{\text {hydro }}$ producing a stable 
$C F$ regime and (c) at higher cathodic counter-pressure (for example $\Delta P_{\text {hydro }}=4$ bars), the profile is pushed towards nanoslit entrance producing a stacking CS regime.

Our simulations have also shown that a stable CF frontline can be obtained in a sharp transition region of surface charge, between propagating $\mathrm{CF}$ at high surface charge and stacking CS located at the nanochannel entrance at low surface charge. However, since the range of the two parameters (surface charge and electric field) to obtain this stable CF point is too narrow, only a few experiments have been reported. For example, Hluskou et al [30] have experimentally reported concentration of negatively charged BSA, with a dynamic characteristic of co-ionic species stacking on the enrichment shock, associated to a concentration factor of 100 after 200 seconds.

In order to stabilize the propagating modes at $1 / D_{u} \ll 1$, we have previously proposed a pressure-assisted preconcentration protocol in which a hydrodynamic pressure is added to reduce (or enhance) the electroosmotic flow. This strategy has allowed a better control of the preconcentration frontline location[31]. Here, we will show that such low cathodic counterpressure allows stabilization of the $\mathrm{CF}$ frontline on the enrichment shock (see Fig 1.b)). In the first part of the paper, pressure-assisted electropreconcentration of fluorescein sodium as a model anionic analyte will be presented. The role of the nanoslit length in the competition between electrophoretic velocity and the BGE velocity at the CP enrichment shock will be discussed based on experiments with different nanoslit lengths. The three different regimes of preconcentration (propagating CF -Fig.1a), stable CF - Fig.1b) and CS - Fig.1c)) observed at different electric field E and counter-pressure $\mathrm{P}$ can be reported on "E/P" diagram. We will show how this diagram varies as a function of the nature of $\mathrm{BGE}(\mathrm{KCl}$ and $\mathrm{NaCl})$ at low ionic strength. Finally, to evaluate the ability of such pressure-assisted protocol to concentrate negatively charged proteins, experiments 
were realized with ovalbumin in HEPES. The E/P diagram for ovalbumin confirms the existence of a large range of parameters where a stable CF regime is obtained.

\section{The role of the counter-pressure on the stabilization of the frontline}

Pressure-assisted electro-preconcentration consists in applying an external hydrodynamic pressure $\Delta \mathrm{P}_{\text {hydro }}$ that produces an additional flow called $\mathrm{J}_{\text {pressure }}=\mathrm{J}_{\mathrm{P}}$ through the nanochannel. In addition to electroosmotic $\left(\mathrm{J}_{\mathrm{EOF}}\right)$ and electrophoretic $\left(\mathrm{J}_{\mathrm{EP}}\right)$ flows that are competing through the $\mathrm{CP}$ effect, this additional hydrodynamic flow $\left(\mathrm{J}_{\mathrm{P}}\right)$ enables to shift the location of the analyte focal point in a direction that depends on the direction of the applied pressure [31]. The total flow is given by $\mathrm{J}=\mathrm{J}_{\mathrm{EOF}}+\mathrm{J}_{\mathrm{EP}}+\mathrm{J}_{\mathrm{P}}$ where the additional hydrodynamic flow $\mathrm{J}_{\mathrm{P}}$ can take positive or negative values. In the absence of a hydrodynamic pressure $\left(\mathrm{J}_{\mathrm{P}}=0\right)$, we will use the notations "classical" or "conventional" to describe electropreconcentration.

Let's first describe classical electropreconcentration with a model BGE such as $\mathrm{KCl}$ and $\mathrm{NaCl}$. At low ionic strength $\mathrm{C}_{\mathrm{BGE}}=10 \mu \mathrm{M}$ and $v_{2}^{*}<1$, a propagating frontline should be observed in the cathodic reservoir (Fig.1a). Zangle's theory allows predicting the location of the preconcentration frontline by comparing the nondimensionalized velocity of the analyte $v_{i}^{*}$ to the critical values $v_{i}^{* \text { crit }}$ for each region (nanochannel, enrichment zone and cathodic reservoir) [11]. When CP propagates, analytes can move inwards on either side of the enrichment shock if their mobility is low enough to travel in the direction of the bulk flow in the enrichment zone but high enough to travel against bulk flow in the reservoir following the condition: $v_{i, e}^{* \text { crit }}>v_{i}^{*}>v_{i, c r}^{* \text { crit }}$. Both critical velocities in the enrichment zone and in the cathodic reservoir are respectively $v_{i, e}^{*}$ crit $=v_{2}^{*}$ and $v_{i, c r}^{* \text { crit }}=1 / D_{u}$. Supporting information SI.1 gives calculations of $1 / D_{u}$ and $v_{2}^{*}$ for both $\mathrm{KCl}$ and $\mathrm{NaCl}$ as $\mathrm{BGE}$, and shows that the condition $v_{i, e}^{* \text { crit }}>v_{i}^{*}>v_{i, c r}^{* \text { crit }}$ is always respected in 
our experiments, predicting a stacking of fluorescein at the enrichment shock interface in the cathodic reservoir [11].

We describe now how pressure-assisted electropreconcentration will modify the propagating frontline. In the case of an additional pressure applied from the cathode to the anode (named "cathodic pressure"), such cathodic counter-pressure reduces the role of EOF flow by promoting the electrophoretic component. Increasing $J_{P}$ at moderate values of pressure allows stabilization of the CF profile as function of time as described in Figure 1b). Such stable CF electropreconcentration regime is usable for applications and can produce high concentration rate whose value increases with time. At higher counter-pressure, the analyte is pushed towards the entrance of the nanochannel in a cathodic stacking CS regime (Fig.1c)).

\section{Materials and methods}

Glass chip fabrication including glass microstructuration and bonding has been previously extensively described by A-C. Louër et al. [31]. 
a)
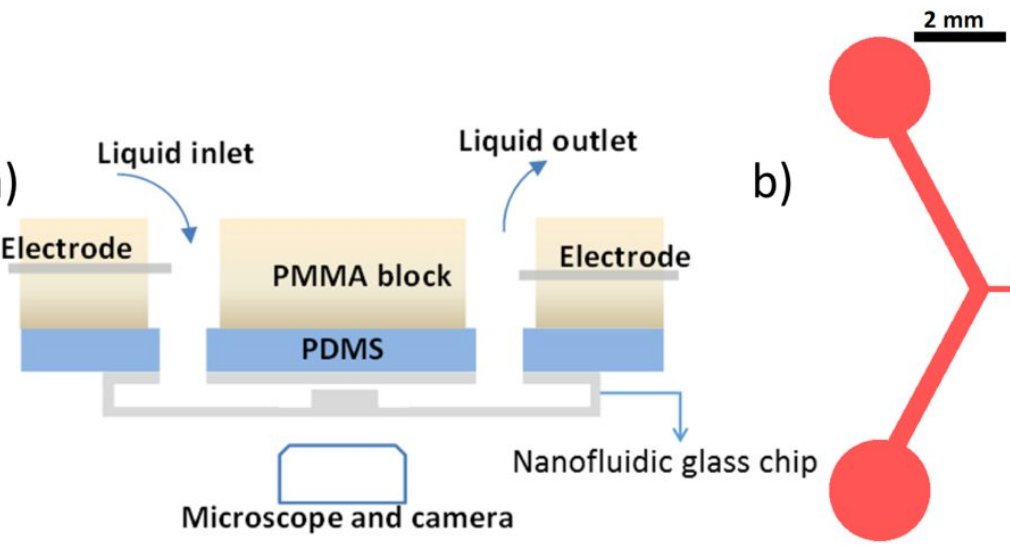

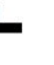

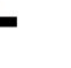

Central part Top view

(1)

Microscope and camera

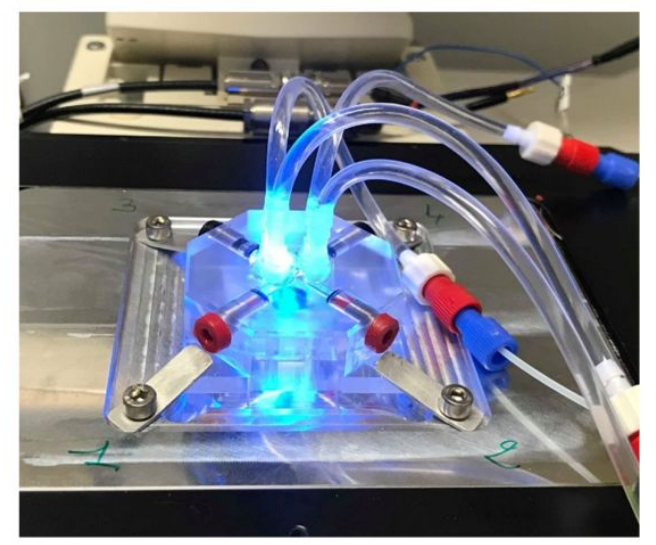

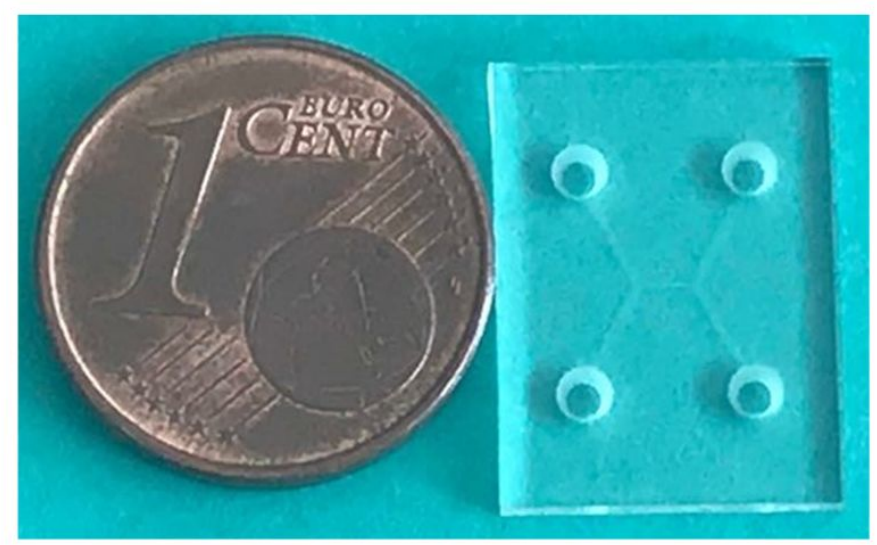

Figure 2. a) schematic of the PMMA chip holder allowing mounting the nanofluidic chip on the inverted microscope and picture of the holder recorded during experiment, b) schematic of the $H$ chip with an integrated nanochannel and top-view photo of one nanofluidic glass chip.

Briefly, in the center of the " $\mathrm{H}$ " type $1 \mathrm{~cm}$ long chip, the nanoslit has a width $w$ of $100 \mu \mathrm{m}$, a height $h_{n}$ of $150 \mathrm{~nm} \pm 10 \mathrm{~nm}$ and, depending of experiments, a length $\mathrm{L}$ of $500 \mu \mathrm{m}$ or $100 \mu \mathrm{m}$ (Figure 2). Both anodic and cathodic reservoirs are $1.5 \mu \mathrm{m}$ deep.

For preconcentration experiments, fluorescein sodium salt from Sigma Aldrich was diluted at $10 \mu \mathrm{M}$ in $\mathrm{KCl}$ or $\mathrm{NaCl}$, whereas Texas red conjugated ovalbumin from Life Technologies was diluted in a $10 \mu \mathrm{M}$ HEPES buffer. The external voltage was generated by a DC Keithley 238 power supply associated with a Keithley 7001 multiplexer. The additional hydrodynamic pressure was 
applied through an ELVEFLOW® OB1 pressure generator, while sequential injection and flow stop were achieved with an ELVEFLOW® Multiplexer. Two cleaning processes were done before each experiment. First, each new chip was cleaned using the same protocol consisting in successive channels rinses with different solutions: 10 minutes with $1 \mathrm{M}$ sodium hydroxide $(\mathrm{NaOH})$ solution, 10 minutes with $0.1 \mathrm{M} \mathrm{NaOH}$ solution, 15 minutes with deionized water and 2 hours with the buffer solution. To get the same initial state before each application of electric field and pressure, an electro-cleaning was used based on three steps: i) applying $20 \mathrm{~V}$ for 2 minutes, ii) injection of the buffer for 10 minutes to renew the solution into the device, and finally iii) applying pressure in the four reservoirs for 10 minutes. The analyte solution is then injected with a syringe, in the four reservoirs. For imaging, an inverted fluorescent microscope (Axiovert 200, Zeiss), a mechanical shutter (Uniblitz VCMD1) and a CCD camera (Orca-ER, Hamamatsu) were used. Images were captured at regular intervals during preconcentration experiments ( 10 seconds during the first minute and then every 60 seconds). A Matlab ${ }^{\circledR}$ program was developed first to monitor the fluidic set-up during experiments and then to extract and analyze fluorescence profiles based on a preliminary calibration [31].

\section{Results and discussion}

\subsection{Pressure-assisted electro-preconcentration of fluorescein: stabilization of CF regime:}

The BGE concentration was kept at $10 \mu \mathrm{M}$ in all experiments to maintain the same CP buffer effect with $1 / D_{u}=0.15$ and $v_{2}^{*}=0.83$ for $\mathrm{KCl}$ and $1 / D_{u}=0.18$ and $v_{2}^{*}=0.83$ for $\mathrm{NaCl}$. In order to study how both electric field E and pressure $\mathrm{P}$ can affect the stabilization of the CF regime, experiments with fluorescein have been performed at different external fields, E ranging from $5 \mathrm{~V} / \mathrm{cm}$ up to $80 \mathrm{~V} / \mathrm{cm}$, and at several additional cathodic counter-pressures, $\mathrm{P}$, ranging from 0 to 0.4 bars. Two different nanoslit lengths $(\mathrm{L}=500 \mu \mathrm{m}$ and $\mathrm{L}=100 \mu \mathrm{m})$ have been studied. 


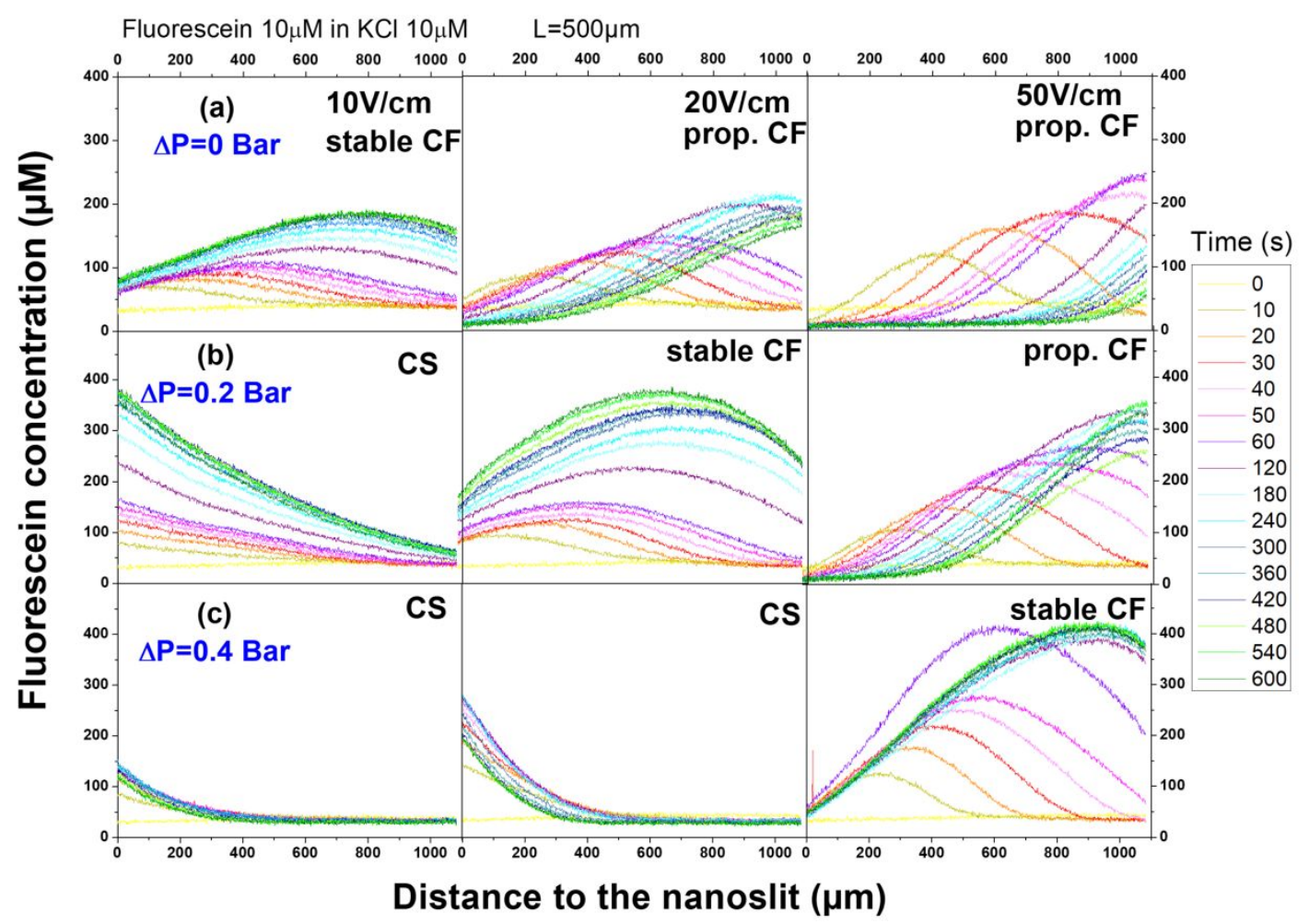

Figure 3. Electropreconcentration profiles of fluorescein diluted in $\mathrm{KCl}$ recorded for a nanoslit with $L=500 \mu \mathrm{m}$ at several additional hydrodynamic pressures (a) $\Delta P=0$ (conventional preconcentration), (b) $\Delta P$ $=0.2$ bars and (c) $\Delta P=0.4$ bars.

As shown in Figure 3a) for conventional cathodic preconcentration without any hydrodynamic counter-pressure $\left(\mathrm{J}_{\mathrm{P}}=0\right)$, a stable CF profile is observed at low field $(10 \mathrm{~V} / \mathrm{cm})$, whereas an expected propagating CF is observed at higher fields $20 \mathrm{~V} / \mathrm{cm}$ and $50 \mathrm{~V} / \mathrm{cm}$. One should note that the higher the field $\mathrm{E}$ is, the more propagating is the profile inside the cathodic reservoir. These experimental observations agree with the analytical calculations of Santiago [9-10] and Zangle [11], as well as with our previous simulations [12] at very low ionic strength, which both predict the cathodic focusing $(\mathrm{CF})$ profile to be unstable over time especially for high electric fields and high surface charge. 
Applying the cathodic counter-pressure $\Delta \mathrm{P}$ allows stabilizing the fluorescein concentration profile as it can be observed in Figures $3 \mathrm{~b}$ ) and $3 \mathrm{c}$ ). For example, at $20 \mathrm{~V} / \mathrm{cm}$ and $\Delta \mathrm{P}=0.2$ bar (Fig.3b)), the maximum of fluorescence intensity occurring at a distance of $600 \mu \mathrm{m}$ from the nanoslit entrance remains quite constant as function of time even after 3 minutes. Similarly, at $50 \mathrm{~V} / \mathrm{cm}$ and $\Delta \mathrm{P}=0.4$ bar (Fig.3c)), after 60 seconds, the $\mathrm{CF}$ peak stabilizes at a larger distance from the nanoslit, namely $950 \mu \mathrm{m}$. Also, the concentration does not decrease for longer times which reflects a stable CF regime. This result shows that an additional hydrostatic pressure can be used to tune the buffer CP effect. This influence of additional flows on CP effect was also commented by Wang et al. [32] who has observed a propagating $\mathrm{CP}$ in their structures, while a stable CP region could be obtained when adding a transverse EOF flux in their "H shape" device. At low electric field, adding a high counter-pressure seems to constrain the preconcentration front toward the nanoslit in the same direction as the additional hydrostatic flow. At $10 \mathrm{~V} / \mathrm{cm}$ and $\Delta \mathrm{P}=0.2$ bar (Fig.3b)), or $20 \mathrm{~V} / \mathrm{cm}$ and $\Delta \mathrm{P}=0.4$ bar (Fig.3c)), the profile is stacked at the entrance of the nanoslit producing a CS regime. Similar experiments have been performed with chips that integrate shorter nanoslit $(\mathrm{L}=100 \mu \mathrm{m}$ - results not shown here - see Figure 6 in the following section). To conclude, adding a hydrodynamic counter-pressure from the cathodic side during fluorescein preconcentration allows manipulation and stabilization in space of the preconcentration frontline.

\subsection{Time dependence of the localization of the preconcentration frontline:}

Analyzing the evolution as function of time of both the peak location $d_{\max }$ and its corresponding concentration $\mathrm{C}_{\max }$ appears also of great interest to determine the concentration rate $\mathrm{C}_{\max } / \mathrm{C}_{0}$ and thus check the efficiency of the nanodevice to concentrate the analyte. Also, it allows checking the stability of the CF regime for intermediate values of counter-pressure. Figure 4 shows such an analysis of the experiments previously reported in Figure 3, for a nanoslit length $\mathrm{L}=500 \mu \mathrm{m}$. 


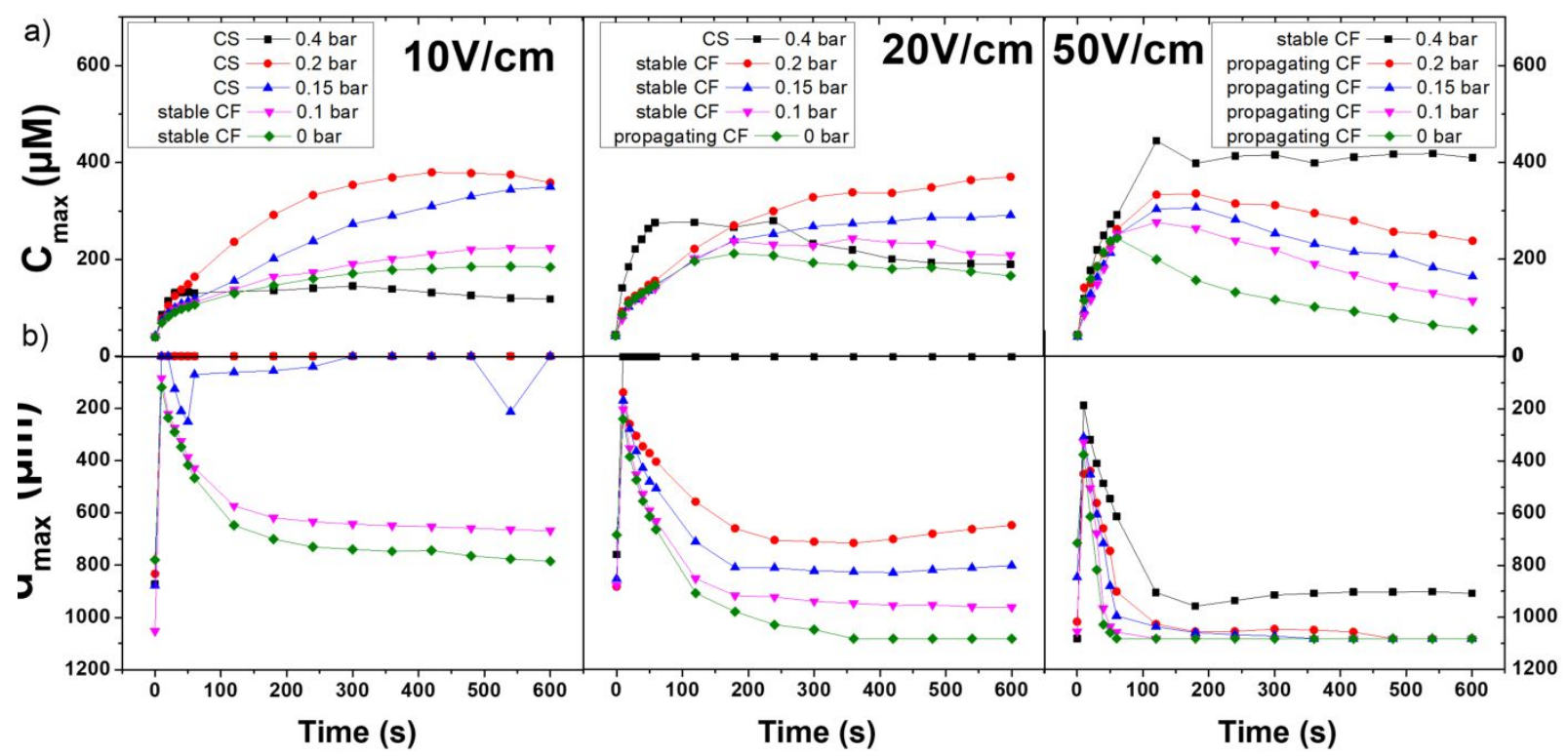

Figure 4. a) Evolution with time of $C_{\max }$ the maximum concentration of each profile for $10 \mathrm{~V} / \mathrm{cm}, 20 \mathrm{~V} / \mathrm{cm}$ and 50V/cm given in Figure 3, and b) corresponding time dependence of its location (distance from the nanoslit) for $10 \mathrm{~V} / \mathrm{cm}, 20 \mathrm{~V} / \mathrm{cm}$ and $50 \mathrm{~V} / \mathrm{cm}$. A critical time of $200 \mathrm{~s}$ is observed to reach the stabilization.

As shown in Figure $4 \mathrm{a}$ ) for $\mathrm{E}=20 \mathrm{~V} / \mathrm{cm}$, adding a counter-pressure $\Delta \mathrm{P}$ in the range $0.1-0.2$ bar allows stabilization of the CF regime with a fixed localization at time higher than 200 seconds. One can observed a shift of the peak location from around $900 \mu \mathrm{m}$ to $600 \mu \mathrm{m}$ when $\Delta \mathrm{P}$ increases from 0.1 to 0.2 bars. The optimal counter-pressure $\Delta \mathrm{P}_{\mathrm{opt}}=0.2$ bars produces the most stable $\mathrm{CF}$ regime with a concentration that increases with time to reach $\mathrm{C}_{\max }=450 \mu \mathrm{M}$ after 10 minutes $\left(\mathrm{C}_{\text {max }} / \mathrm{C}_{0}=45\right)$. At higher pressure $(\Delta \mathrm{P}=0.4$ bars $)$ the profile is stacked at the entrance of the nanoslit producing a CS regime as observed in Fig4.b) (dark curve). As shown in figure 4a), the higher the electric field is, the higher the optimal $\Delta \mathrm{P}_{\text {opt }}$ is too with $\Delta \mathrm{P}_{\text {opt }}=0.1$ bars for $\mathrm{E}=10 \mathrm{~V} / \mathrm{cm}$, $\Delta \mathrm{P}_{\mathrm{opt}}=0.2$ bars for $\mathrm{E}=20 \mathrm{~V} / \mathrm{cm}$ and $\Delta \mathrm{P}_{\mathrm{opt}}=0.4$ bars for $\mathrm{E}=50 \mathrm{~V} / \mathrm{cm}$. 
However, since both fields $20 \mathrm{~V} / \mathrm{cm}$ and $50 \mathrm{~V} / \mathrm{cm}$ produce the same concentration rate $\mathrm{C}_{\max } / \mathrm{C}_{0}$ of about 45 , the best conditions of preconcentration using $\mathrm{KCl}$ as $\mathrm{BGE}$ are $20 \mathrm{~V} / \mathrm{cm}$ and $\Delta \mathrm{P}_{\mathrm{opt}}=0.2$ bars if one wants to minimize both $\mathrm{E}$ and $\mathrm{P}$. However, it appears that the time transition to reach stabilization of the CF regime is shorter at $50 \mathrm{~V} / \mathrm{cm}$ as shown by the dark curves of figures $4 \mathrm{a}$ ) and b) corresponding to 0.4 bar. The highest the field is, the higher P has to be fixed to stabilize the preconcentration frontline. The concentration rate reaches a limit as function of field since the enrichment shock is a weak shock due to relatively low electric fields across the interface between enrichment region and cathodic reservoir [11].

It is of great interest to study how the nature of the BGE can affect these preconcentration profiles. Similar experiments were done with $\mathrm{NaCl}$ as $\mathrm{BGE}\left(1 / D_{u}=0.18\right.$ and $v_{2}^{*}=0.83$-see SI. 1 for the calculations) for which a propagating regime is also predicted. With a higher inverse Dukhin number $1 / D_{u}$ for $\mathrm{NaCl}$ compared to $\mathrm{KCl}$, on should expect an easier stabilization of the propagating $\mathrm{CF}$ in this $\mathrm{BGE}$. 
Figure 5 shows how both the peak location $\mathrm{d}_{\max }$ and the maximum in concentration $\mathrm{C}_{\max }$ evolve with time. Stabilization of the CF regime appears at lower electric fields, namely $5 \mathrm{~V} / \mathrm{cm}$ and $10 \mathrm{~V} / \mathrm{cm}$, compared to $\mathrm{KCl}$ experiments. One can also observed the optimal $\Delta \mathrm{P}_{\mathrm{opt}}$ with $\Delta \mathrm{P}_{\mathrm{opt}}=$ 0.15 bars for $\mathrm{E}=5 \mathrm{~V} / \mathrm{cm}$ and $\Delta \mathrm{P}_{\text {opt }}=0.2$ bars for $\mathrm{E}=10 \mathrm{~V} / \mathrm{cm}$. For these optimal conditions, the localisation of the focal point ranges between $450 \mu \mathrm{m}$ and $500 \mu \mathrm{m}, 100 \mu \mathrm{m}$ closer to the nanoslit entrance than for $\mathrm{KCl}$ experiments. In addition, the concentration rate $\mathrm{C}_{\max } / \mathrm{C}_{0}$ is largely enhanced with $\mathrm{C}_{\max } / \mathrm{C}_{0}=130$ for $\mathrm{E}=5 \mathrm{~V} / \mathrm{cm}-\Delta \mathrm{P}_{\mathrm{opt}}=0.15$ bars (blue curve in Figure 5.a)) and $\mathrm{C}_{\max } / \mathrm{C}_{0}=120$ for $\mathrm{E}=10 \mathrm{~V} / \mathrm{cm}-\Delta \mathrm{P}_{\mathrm{opt}}=0.2$ bars (red curve in Figure 5.b)). All these results confirm that stabilization of the propagating $\mathrm{CF}$ regime is easier in $\mathrm{NaCl}$. This is not surprising since our analyte sodium 
fluorescein diluted at $10 \mu \mathrm{M}$ contains as many $\mathrm{Na}^{+}$cations as the background electrolyte $(\mathrm{NaCl}-$ $10 \mu \mathrm{M})$. Even if potassium can also exchange with sodium counter-ion in the $\mathrm{KCl} \mathrm{BGE}, \mathrm{NaCl}$ electrolyte appears here as a better BGE for stabilization of CF regime at low voltage. Looking at $10 \mathrm{~V} / \mathrm{cm}$ field and comparing left part of figure 4 and figure $5 \mathrm{~b}$ ), it appears that the $\mathrm{NaCl} \mathrm{BGE}$ leads to stable $\mathrm{CF}$ until 0.15 bar associated to a higher $\mathrm{C}_{\max } / \mathrm{C}_{0}$ preconcentration factor compared the $\mathrm{KCl} \mathrm{BGE}$. We assume that the sodium cations provide rapid exchanges between the $\mathrm{NaCl} \mathrm{BGE}$ and the fluorescein. This situation offers a narrower and more fluorescent $\mathrm{CF}$ stable window in the preconcentration diagram. With $\mathrm{KCl} \mathrm{BGE}$, the bigger and slower potassium counter-ion will slightly change the polarisation of the fluorescein and it probably creates two distinct populations of fluorescein the first majority one with $\mathrm{Na}^{+}$and the other one with $\mathrm{K}^{+}$. This configuration leads to broader but less fluorescent preconcentration plug during the CF because of the two distinct electrophoretic mobilities coming from $\mathrm{K}^{+}$-fluorescein and from the $\mathrm{Na}^{+}$-fluorescein.

\section{3 "Electric field/Pressure" diagrams:}

From all these experimental results at a unique ionic strength of $10 \mu \mathrm{M}$, it is of great interest to build "electric field / pressure" diagrams, which were obtained in the following way. For each experiment, a classification of the different types (propagating $\mathrm{CF}$, or stable $\mathrm{CF}$, or $\mathrm{CS}$ ) of preconcentration regimes obtained can be done. For each profile obtained, a point corresponding to one of the three regimes is located in an "electric field / pressure" diagram that will be named E/P diagram in the following. 
a) $\mathrm{L}=500 \mu \mathrm{m}$
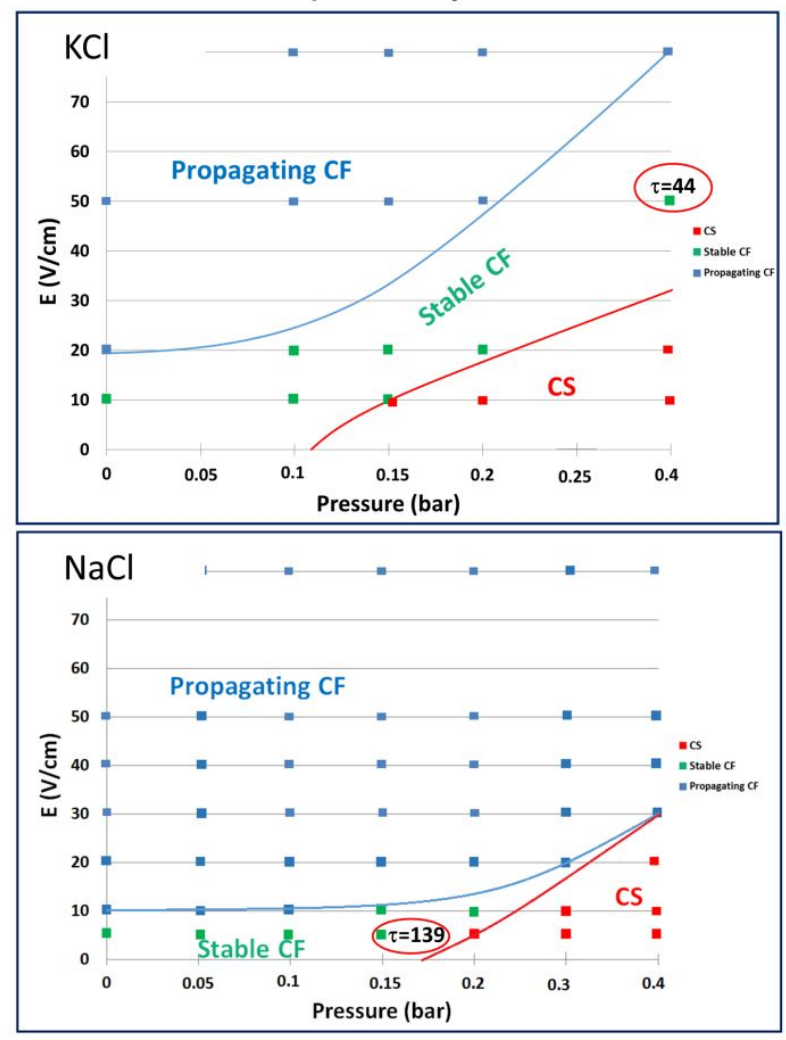

b) $L=100 \mu \mathrm{m}$
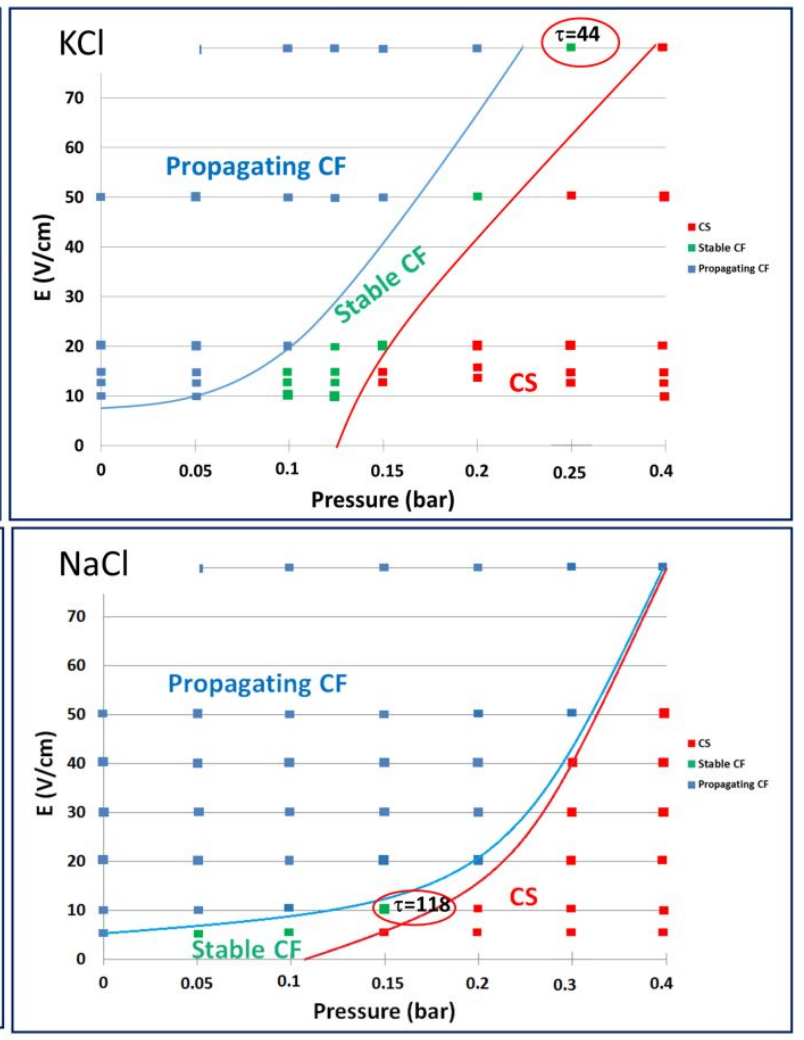

Figure 6. The "Electric field /Pressure" diagram established from Fluorescein electropreconcentration experiments using a chip that integrates a 150nm-high nanoslit with a length (a) $L=500 \mu \mathrm{m}$ or (b) $L=100 \mu \mathrm{m}$ and for the two $\mathrm{BGE} \mathrm{KCl}$ and $\mathrm{NaCl}(\mathrm{C}=10 \mu \mathrm{M})$. Blue and red curves are guidelines to evidence the limit between each regime. The maximal value of the preconcentration rate $\tau=C_{\max } / C_{0}$ is also reported in each diagram showing for each diagram the best couple of parameters $(E, P)$.

These E/P diagrams allow observing the three regions that correspond to the three different preconcentration regimes, namely propagating CF (in blue), stable CF (in green) and CS (in red). Four E/P diagrams have been obtained for two different lengths of the nanoslit $\mathrm{L}_{\text {nanoslit }}=500 \mu \mathrm{m}$ (see Figure 6.a)) and $\mathrm{L}_{\text {nanoslit }}=100 \mu \mathrm{m}$ (see Figure 6.b)) in the two BGE solutions, $\mathrm{KCl}$ and $\mathrm{NaCl}$. In these diagrams, the maximal value of the preconcentration rate $\tau=\mathrm{C}_{\max } / \mathrm{C}_{0}$ has also been reported allowing a direct observation of the best couple $(\mathrm{E}, \mathrm{P})$ of parameters. 
Whatever the nature of the buffer, it is observed that the short nanoslit $\mathrm{L}=100 \mu \mathrm{m}$ always leads to propagating regimes when no counter-pressure is added $(\Delta \mathrm{P}=0$ for conventional electropreconcentration). On the other hand, with long nanoslit ( $\mathrm{L}=500 \mu \mathrm{m})$, a stable $\mathrm{CF}$ regime can be obtained at $\Delta \mathrm{P}=0$ and low field, namely $10 \mathrm{~V} / \mathrm{cm}$ for $\mathrm{KCl}$ and $5 \mathrm{~V} / \mathrm{cm}$ for $\mathrm{NaCl}$. This is explained by a more pronounced CP effect for $500 \mu \mathrm{m}-$ long nanoslit. Moreover, the comparison between the diagrams established for $\mathrm{KCl}$ and for $\mathrm{NaCl}$ shows that the area of observation of the stable CF regime (in green) is shifted towards the weak electric fields for $\mathrm{NaCl}$. Preconcentration rates obtained are also greater with $\mathrm{NaCl}$ than with $\mathrm{KCl}$, as shown by the values circled in red on the 4 diagrams. With $\mathrm{NaCl}$ as background solution, it is therefore easy to stabilize $\mathrm{CF}$ regime at low electric field $(<$ or $=$ at $5 \mathrm{~V} / \mathrm{cm})$ and low counter-pressure $(<$ or $=$ at 0.15 bar $)$. These conditions produce the maximal value of the preconcentration rate $\tau=\mathrm{C}_{\max } / \mathrm{C}_{0} \sim 140$. The analyst will thus have to choose between a broader stability window or a high preconcentration factor. This choice will be driven by the instrumentation, namely the speed of the camera or the sensitivity of the optical sensor. The initial concentration of the analyte is also another main parameter to consider before choosing the nature of the BGE. For trace analysis, a fluorescence optical sensor with high spatial resolution will be probably preferred since high preconcentration rate is required. In that case, $\mathrm{NaCl}$ appears as the best BGE. On the other hand, with analyte at higher concentration, with $\mathrm{KCl}$ as $\mathrm{BGE}$, the stability of the preconcentration plug could be reached faster because of the wider stability domain (see Figure 6). Once again, these results underline the interdependence between the choice of the BGE and the quality of the analysis by electrophoretic methods. In other words, similarly as the popular free zone electrophoresis, electropreconcentration with a microfluidic device bearing a nanoslit requires cautious adjustments of the BGE composition regarding to the sample composition and its available quantity. 


\subsection{Pressure-assisted electro-preconcentration of Ovalbumin:}

Chicken ovalbumin is a $45 \mathrm{kDa}$ protein that is the major component of egg white. Fluorescent ovalbumin can be used as endocytic tracers [33-35] and to estimate the size of the transport pathways of micro-vessels [36]. It is also used in various research fields such as studying the structure and functionality of serpins, and proteomics (egg yolk ovalbumin is frequently used as a molecular marker for calibration of electrophoresis gels) or immunology frequently used to stimulate an allergic reaction on test subjects [37-38].

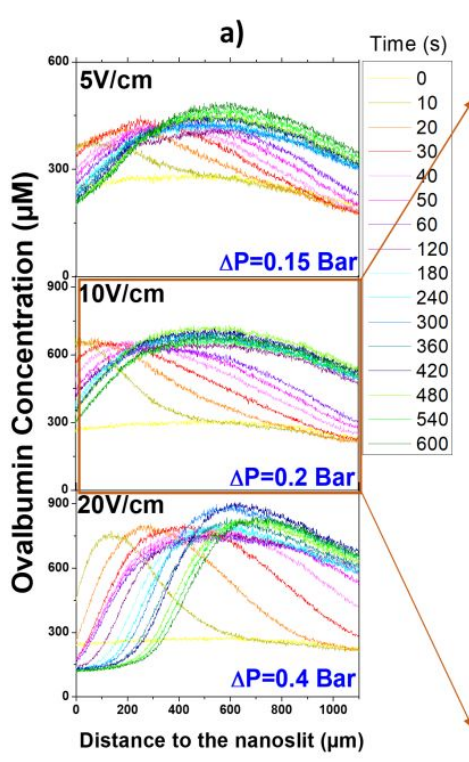

b)

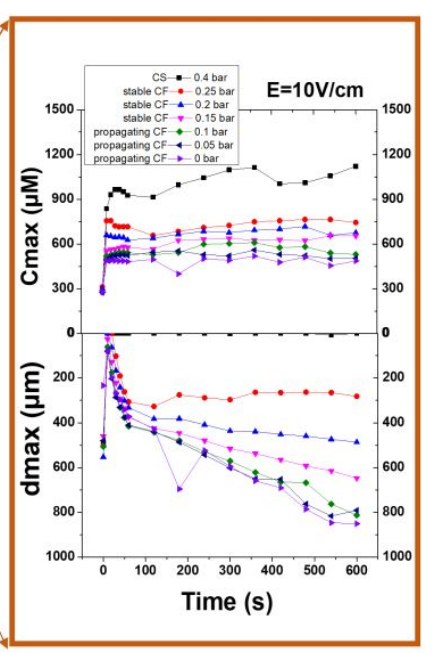

c)

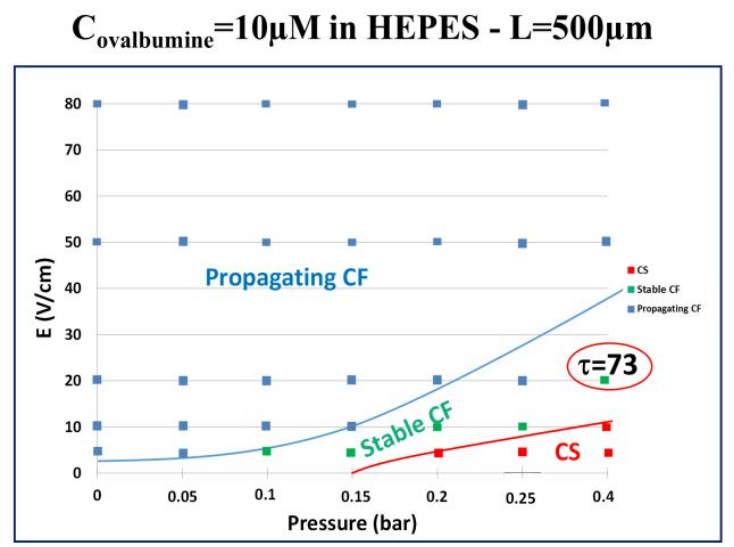

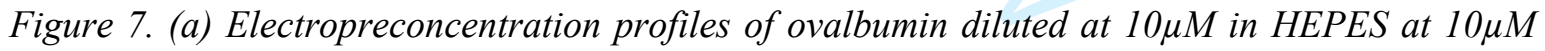
recorded for a nanoslit with $L=500 \mu m$ at different coupled E/P that produce stable CF regimes, (b) Evolution with time of $C_{\max }$ the maximum concentration of each profile for $10 \mathrm{~V} / \mathrm{cm}$ and $0.2 \mathrm{bars}$, and time dependence of its location (distance from the nanoslit), (c) The "Electric field /Pressure" diagram established from ovalbumin electropreconcentration experiments showing at 20V/cm and 0.4 bars the maximal rate $\tau=C_{\max } / C_{0}=73$. 
In order to validate the pressure-assisted protocol for a more applicative protein that our previous model molecule of sodium fluorescein, experiments were carried out with ovalbumin. For these experiments, only $500 \mu \mathrm{m}$-long nanoslit were used as they produce the highest CP effect of the BGE in the MNM structure. Here, the background solution chosen was a real buffer frequently used in analytical chemistry, the organic zwitterionic HEPES compound. Unlike phosphate and bicarbonate buffers, this HEPES buffer solution helps maintaining the structure and function of proteins at low temperatures. Moreover, the use of zwitterionic buffers as BGE allow the application of high separation voltages while maintaining a low current. Ovalbumin was thus diluted at a concentration of $10 \mu \mathrm{M}$ in a $10 \mu \mathrm{M}$ HEPES solution. Ovalbumin was marked with the Texas Red marker whose maximum emission and absorption wavelengths are respectively $\lambda=615 \mathrm{~nm}$ and $596 \mathrm{~nm}$.

Similarly, just as sodium fluorescein, ovalbumin concentrates in the cathodic reservoir. For clarity, only profiles corresponding to a stable CF regime have been reported here in Figure 7a). Despite the high CP effect in HEPES due to the long nanoslit $(\mathrm{L}=500 \mu \mathrm{m})$, propagating regimes are always observed using conventional electrophoresis at $\Delta \mathrm{P}=0$. Quite high counter-pressure must be applied, from 0.15 bar (with $\mathrm{E}=5 \mathrm{~V} / \mathrm{cm}$ ) to $0.4 \mathrm{bar}$ (with $\mathrm{E}=20 \mathrm{~V} / \mathrm{cm}$ ) to stabilize the $\mathrm{CF}$ regime. On the other hand, similarly as for our previous experiments with $\mathrm{NaCl}$ as background salt, it is not necessary to apply high electric fields, as shown in the diagram obtained (Figure 7.b). Obtaining the stable CF regime for a protein of interest such as ovalbumin at low-electric field is very interesting in terms of applications. The maximal concentration rate for ovalbumin of about $\tau=\mathrm{C}_{\text {max }} / \mathrm{C}_{0}=73$ after several minutes is also promising. All those experiments carried out with ovalbumin confirm the great interest of adding an additional counter-pressure to obtain quickly 
stable CF regimes over time inside a micro/nano/micro fluidic device able to concentrate such derivatized biomacromolecule at high concentration rate.

\section{Conclusion}

In this work, a pressure-assisted preconcentration protocol in which a hydrodynamic pressure is added to reduce the electroosmotic flow is shown to stabilize propagating modes at $1 / D_{u} \ll 1$. Low cathodic counter-pressure allows stabilization of the $\mathrm{CF}$ frontline on the enrichment shock whereas higher counter-pressure produces CS stacking regime at the nanoslit entrance. The three different preconcentration regimes (propagating $\mathrm{CF}$, stable $\mathrm{CF}$ and $\mathrm{CS}$ ) observed at different electric field $\mathrm{E}$ and counter-pressure $\mathrm{P}$ for fluorescein sodium $(\mathrm{C}=10 \mu \mathrm{M})$ can be reported on an original "E/P" diagram. The role of the nanoslit length in the competition between electrophoretic velocity and the BGE velocity at the $\mathrm{CP}$ enrichment shock can be easily evidenced on the corresponding "E/P" diagram. It is shown that higher P values are needed for CF stabilization with shorter nanoslit $\left(\mathrm{L}_{\text {nanoslit }}=100 \mu \mathrm{m}\right)$ with a weaker $\mathrm{CP}$ effect, compared to longer slit $\left(\mathrm{L}_{\text {nanoslit }}=500 \mu \mathrm{m}\right)$. The stable $\mathrm{CF}$ region in such " $\mathrm{E} / \mathrm{P}$ " diagram is also changing as function of the nature of $\mathrm{BGE}(\mathrm{KCl}$ and $\mathrm{NaCl})$ with an easier stabilization at low $\mathrm{E}$ for $\mathrm{NaCl}$ and higher preconcentration rates. Finally, the ability of such pressure-assisted protocol to concentrate negatively charged proteins has been tested with $10 \mu \mathrm{M}$ ovalbumin in HEPES and the corresponding E/P diagram for ovalbumin confirms the existence of a stable CF regime at low electric field.

\section{Acknowledgments}

All authors would like to thank ANR for the financial support (DIFLUSEL and NANOCODE). 


\section{References}

(1) L. Gervais, N. D. Rooij, and E. Delamarche, Adv. Mater., vol. 23, no. 24, pp. H151-H176, 2011.

(2) H. Virk, S. Kaur, and G. Randhawa, Environ. Int., 27, 359-362, 2001.

(3) J. A. Quinn, J. L. Anderson, W. S. Ho, and W. J. Petzny, Biophys. J., 12, 990-1007, 1972.

(4) Y. Wang, M. Choi, and J. Han, Anal. Chem., 76, 15, 4426-4431, 2004.

(5) A. Asif and D. Chung, Electrophoresis, 26, 3, 668-673, 2005.

(6) Q. Pu, J. Yun, H. Temkin, and S. Liu, Nano Letters, 4, 1099-1103, 2004.

(7) V. Kašicka, Electrophoresis, 41, 10-35, 2020.

(8) A. Plecis, R.B. Schoch, and P. Renaud, Nano Letters 5(6), 1147-1155, 2005.

(9) Ali Mani, Thomas A. Zangle, and Juan G. Santiago, Langmuir 25, 3898-3908, 2009.

(10) Thomas A. Zangle, Ali Mani, and Juan G. Santiago, Langmuir 25, 3909-3916, 2009.

(11) Thomas A. Zangle, Ali Mani, and Juan G. Santiago, Chem. Soc. Rev. 39, 1014, 2010.

(12) A. Plecis, C. Nanteuil, A.-M. Haghiri-Gosnet, and Y. Chen, Anal. Chem., 80, 9542-9550, 2008.

(13) S. J. Kim, Y. Song, and J. Han, Chem. Soc. Rev. 39, 912, 2010.

(14) C.Wang, Y. Wang, Y. Zhou, Z.Q. Wu and X.H. Xia, Anal. Bioanal Chem., 411, 40074016, 2019.

(15) L.M. Fu, H.H. Hou, P.H. Chiu and R.J. Yang, Electrophoresis, 39, 289-310, 2018.

(16) S. A. Hong, Y. J. Kim, S. J. Kim and S. Yang, Biosens. Bioelectron, 107, 103-110, 2018.

(17) S. J. Lee, J. Lee and K. Kim, Anal. Biochem, 557, 13-17, 2018.

(18) J. Choi, S. Baek, H. C. Kim, J-H. Chae, Y. Koh, S. W. Seo, H. Lee and S.J. Kim, BioChip J., 14(1), 100-109, 2020.

(19) C-C Lin, J-L. Hsu and G-B. Lee, Microfluid Nanofluid, 10, 481-511, 2011.

(20) W. Ouyang and J. Han, Proc Natl Acad Sci USA, 116(33), 16240-16249, 2019.

(21) R. Bharaswaj and J.G. Santiago, J. Fluid. Mech. 543, 57, 2005.

(22) J. M. Sustarich, B.D. Storey and S. Pennathur, Phys. Fluids 22, $112003,2010$.

(23) L. Ouyang, Q. Liu, H. Liang, J. Sep. Sci., 40, 789-797, 2017.

(24) D. W. Inglis, E. M. Goldys, and N. P. Calander, Angew. Chem. Int. 50, 7546-7550, 2011.

(25) W.L. Hsu, D.J. Harvie, M.R. Davidson, H. Jeong, E.M. Goldys and D.W. Inglis, Lab. Chip 14, 3539, 2014.

(26) W.L. Hsu, D.W. Inglis, M. A. Startsev, E. M. Goldys, M.R. Davidson, Anal. Chem., 86, 8711-8718, 2014.

(27) W. Ouyang, Z. Li, J. Han, Anal. Chem., 90(19), 11366-11375, 2018

(28) M.A. Startsev, M. Ostrowski, E.M. Goldys and D.W. Inglis, Electrophoresis, 38, 335-

$341,2017$.

(29) M.A. Startsev, D.W. Inglis, M.S. Baker and E.M. Goldys, Anal. Chem., 85(15), 71337138, 2013.

(30) D. Hlushkou, R. Dhopeshwarkar, R.M. Crooks, U. Tallarek, Lab. Chip 8, 1153-1162, 2008.

(31) A-C. Louër, A. Plecis, A. Pallandre, J-C. Galas, A. Estevez-Torres and A.-M. HaghiriGosnet, Anal. Chem., 85, 79487956-9550, 2013.

(32) Y. Wang, A. Stevens, and J. Han, Anal. Chem., 77, 4293-4299, 2005.

(33) M.S. Swanson and R.R. Isberg, Infect Immun, 64(7), 2585-2594, 1996. 
(34) L.M. Refolo, K. Sambamurti, S. Efthimiopoulous, M.A. Pappolla and N.K. Robakis, J Neurosci Res, 40(5), 694-706, 1995.

(35) G. Grieco, V. Janssens, H.P. Gaide Chevronnay et al., Sci. Rep, 8, 14133, 2018.

(36) J.E. McNamee and M.B. Wolf, Microcirculation, 5(4), 275-280, 1998.

(37) J. Golias, M. Schwarzer, M. Wallner, M. Kverka, H. Kozakova et al. PLOS ONE, 7(5): e37156, 2012.

(38) T. Hilmenyuk, I. Bellinghausen, B. Heydenreich, A. Ilchmann, M. Toda, S. Grabbe, and J. Saloga, Immunology, 129(3), 437-445, 2010. 
a)

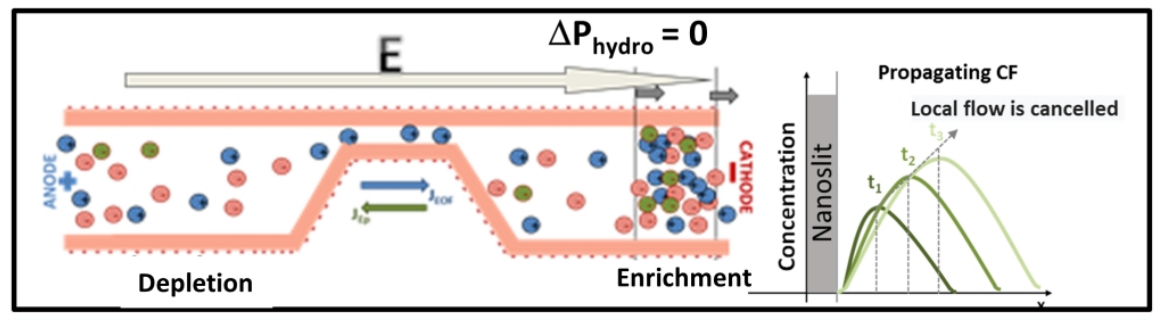

b)

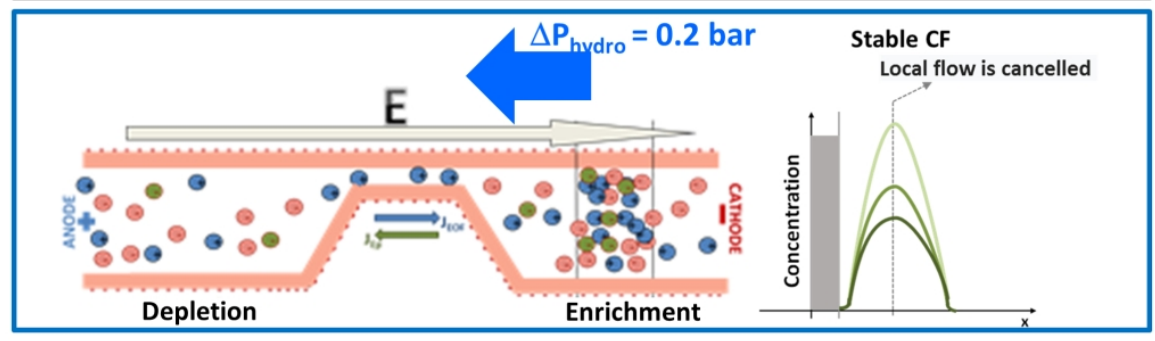

c)

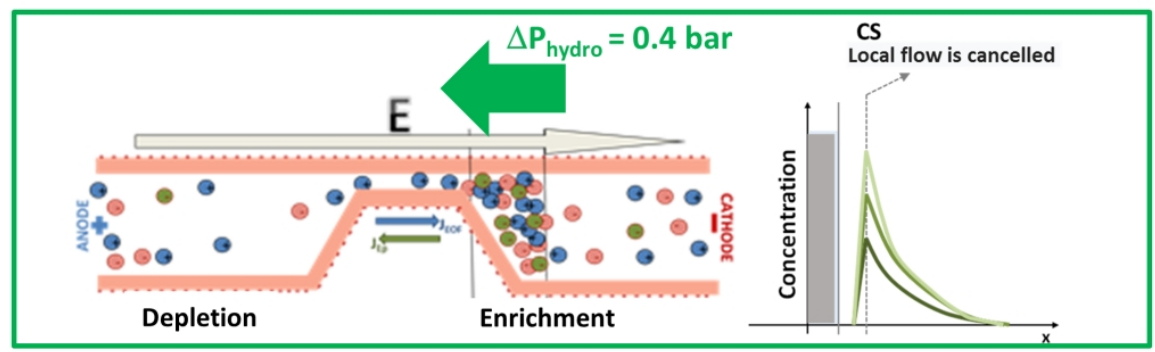

Figure 1. Cross-view of the micro/nano/micro (MNM) device with (a) a typical "propagating CF" profile observed for an anionic analyte stacking and moving at the enrichment shock with $v_{2}{ }^{*}<1$ for conventional electropreconcentration (without any counter-pressure $\Delta P_{\text {hydro }}=0$ ), (b) stabilization of the concentration frontline under the application of a low cathodic counter-pressure $\Delta \mathrm{P}_{\text {hydro }}$ producing a stable $\mathrm{CF}$ regime and

(c) at higher cathodic counter-pressure ( for example $\Delta \mathrm{P}_{\text {hydro }}=4$ bars), the profile is pushed towards nanoslit entrance producing a stacking CS regime 


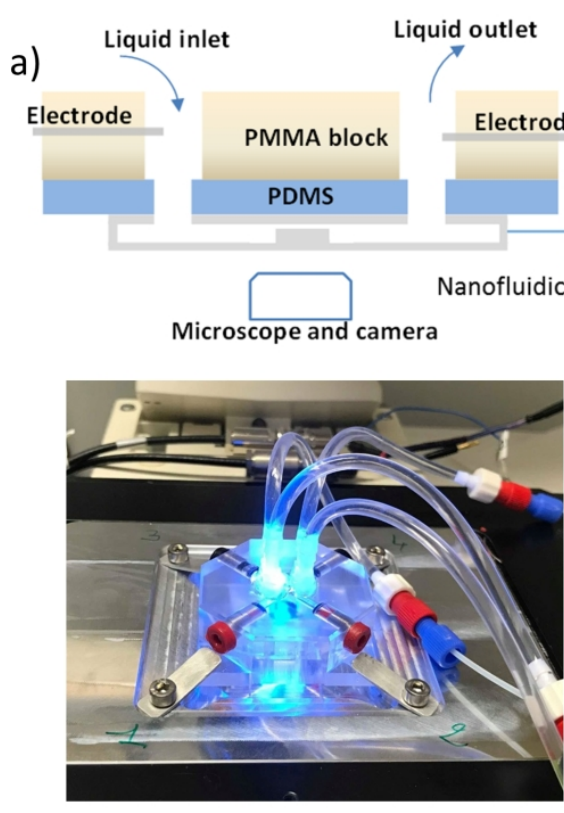

b)
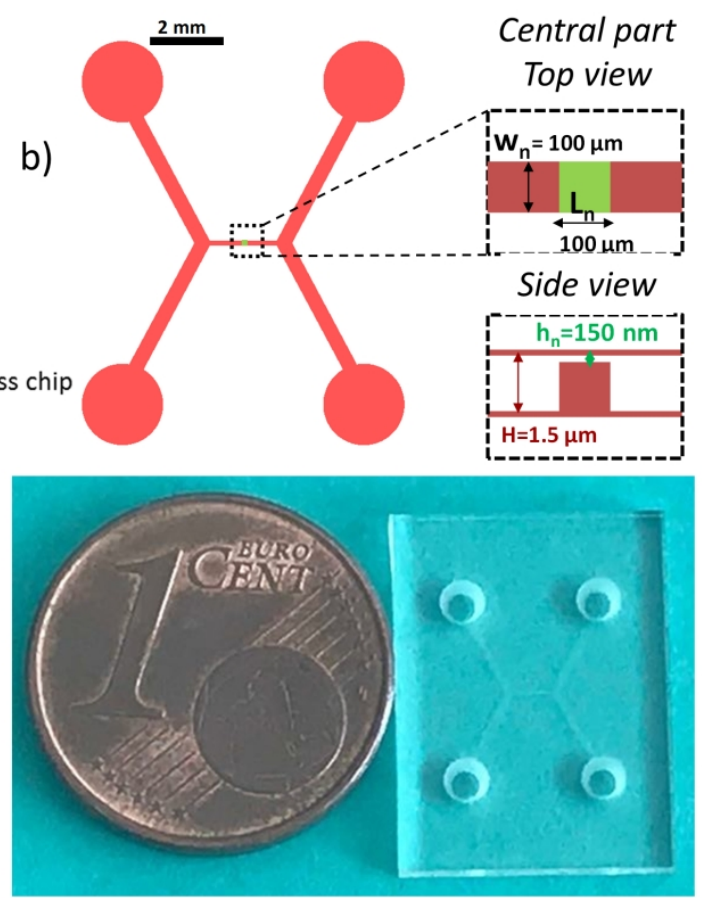

Figure 2. a) schematic of the PMMA chip holder allowing mounting the nanofluidic chip on the inverted microscope and picture of the holder recorded during experiment, $\mathrm{b}$ ) schematic of the $\mathrm{H}$ chip with an integrated nanochannel and top-view photo of one nanofluidic glass chip. 


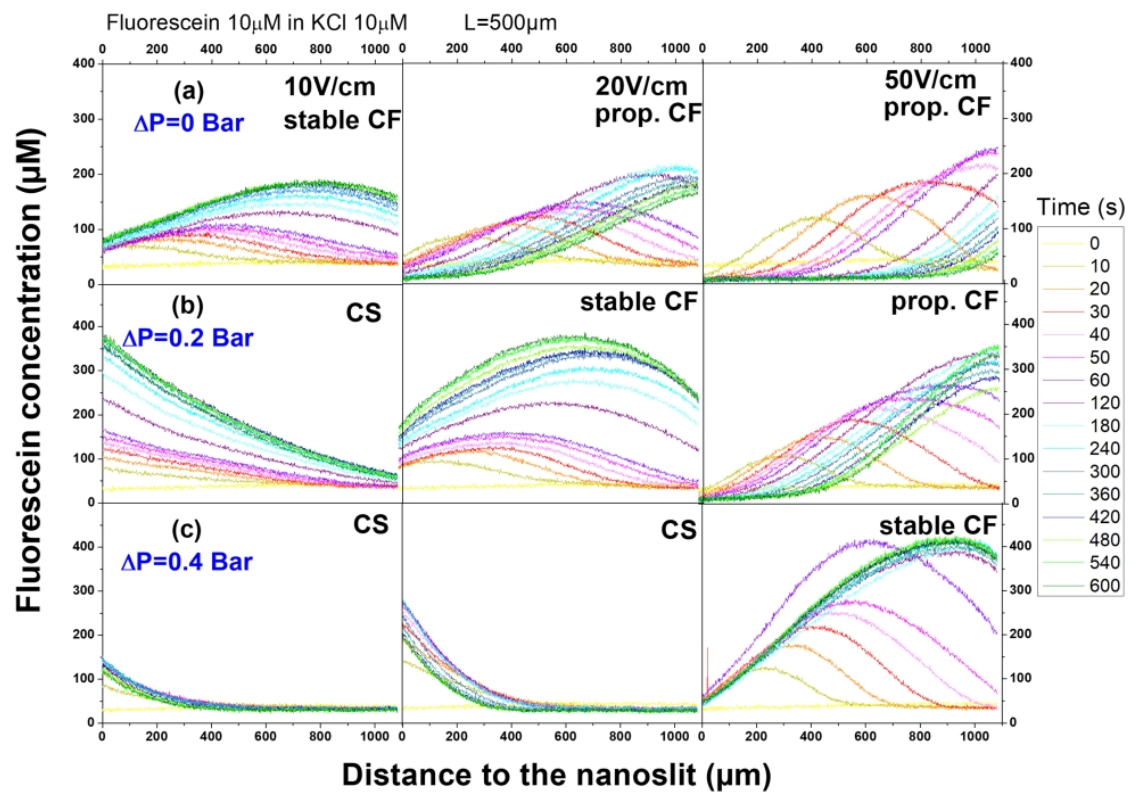

Figure 3. Electropreconcentration profiles of fluorescein diluted in $\mathrm{KCl}$ recorded for a nanoslit with $\mathrm{L}=500 \mu \mathrm{m}$ at several additional hydrodynamic pressures (a) $\Delta \mathrm{P}=0$ (conventional preconcentration), (b) $\Delta \mathrm{P}=0.2$ bars and (c) $\Delta \mathrm{P}=0.4$ bars. 


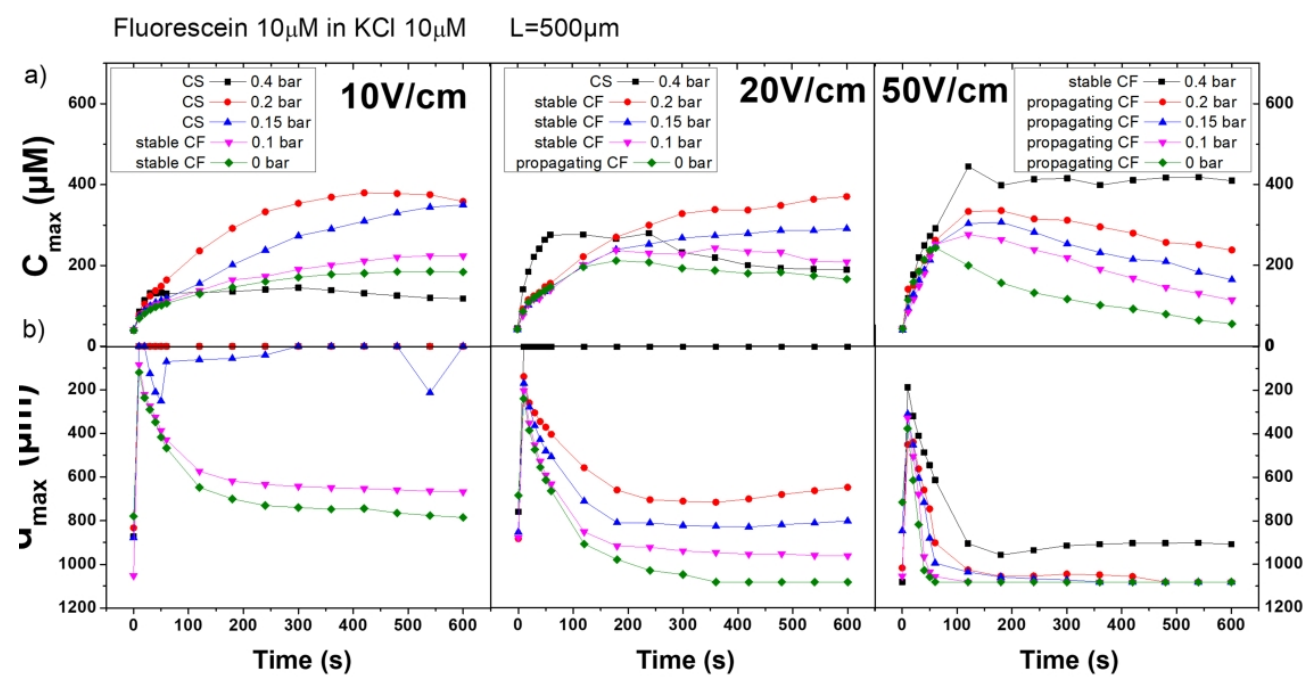

Figure 4. a) Evolution with time of $\mathrm{C}_{\max }$ the maximum concentration of each profile for $10 \mathrm{~V} / \mathrm{cm}, 20 \mathrm{~V} / \mathrm{cm}$ and $50 \mathrm{~V} / \mathrm{cm}$ given in Figure 3, and b) corresponding time dependence of its location (distance from the nanoslit) for $10 \mathrm{~V} / \mathrm{cm}, 20 \mathrm{~V} / \mathrm{cm}$ and $50 \mathrm{~V} / \mathrm{cm}$. A critical time of $200 \mathrm{~s}$ is observed to reach the stabilization. 


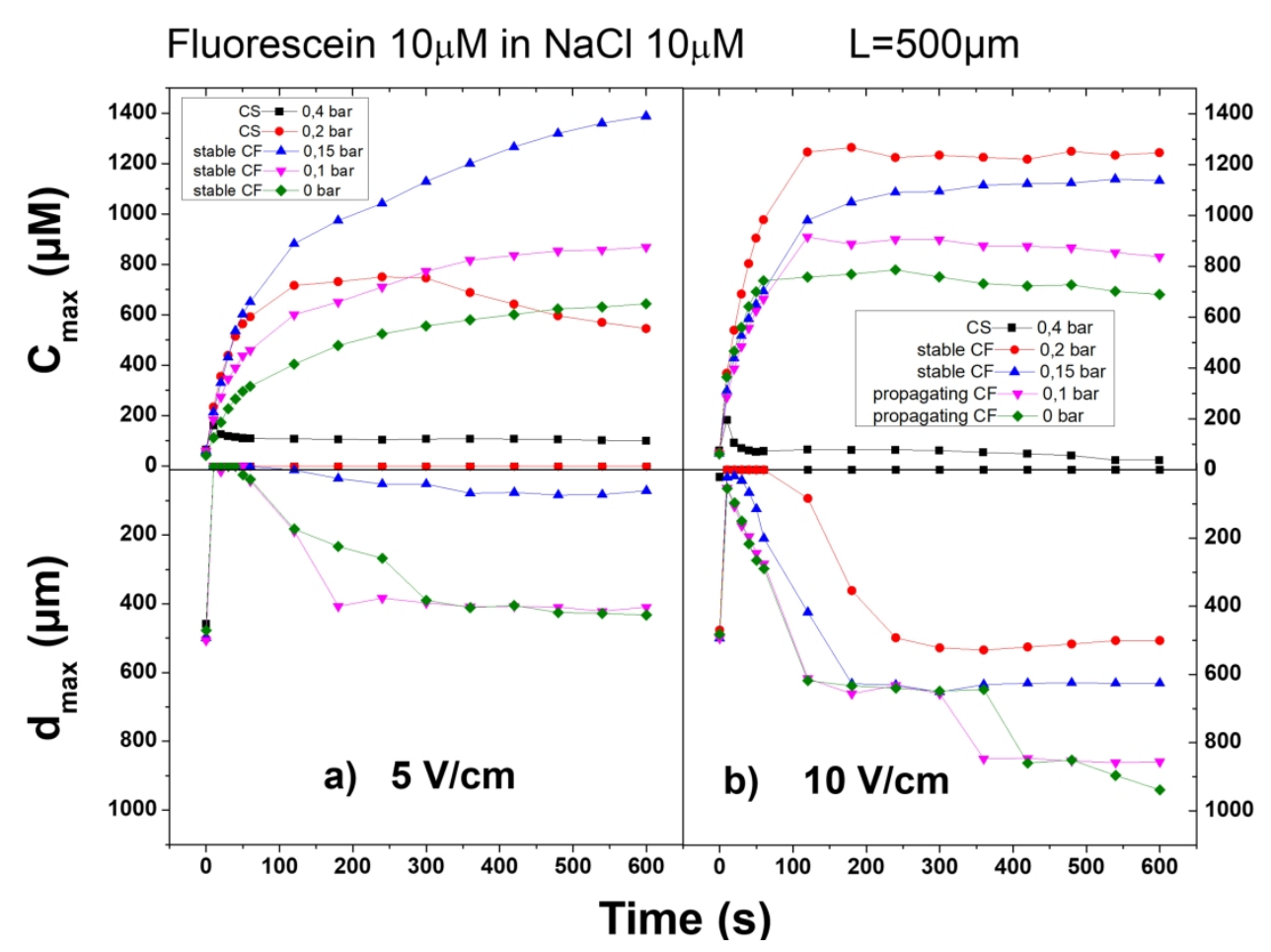

Figure 5. Evolution with time of $C_{\max }$ the maximum concentration of each profile for (a) $10 \mathrm{~V} / \mathrm{cm}$, (b) $20 \mathrm{~V} / \mathrm{cm}$ and (c) $50 \mathrm{~V} / \mathrm{cm}$ for experiments performed in $\mathrm{NaCl}(\mathrm{L}=500 \mu \mathrm{m})$ and time dependence of its location (distance from the nanoslit) for (d) $10 \mathrm{~V} / \mathrm{cm}$, (e) $20 \mathrm{~V} / \mathrm{cm}$ and (f) $50 \mathrm{~V} / \mathrm{cm}$. 
Figure 6. The "Electric field /Pressure" diagram established from Fluorescein electropreconcentration experiments using a chip that integrates a $150 \mathrm{~nm}$-high nanoslit with a length (a) $\mathrm{L}=500 \mu \mathrm{m}$ or (b) $\mathrm{L}=100 \mu \mathrm{m}$ and for the two $\mathrm{BGE} \mathrm{KCl}$ and $\mathrm{NaCl}(\mathrm{C}=10 \mu \mathrm{M})$. Blue and red curves are guidelines to evidence the limit between each regime. The maximal value of the preconcentration rate $\mathrm{T}=\mathrm{C}_{\mathrm{max}} / \mathrm{C}_{0}$ is also reported in each diagram showing for each diagram the best couple of parameters $(E, P)$. 


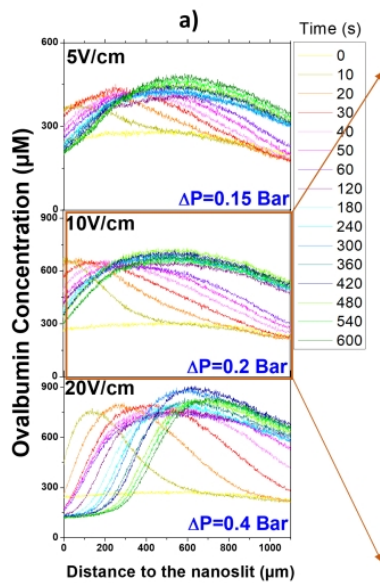

b)

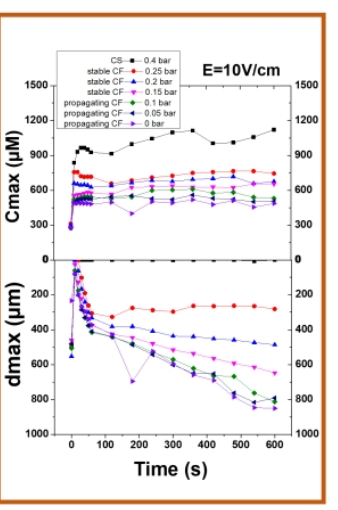

c)

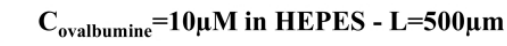

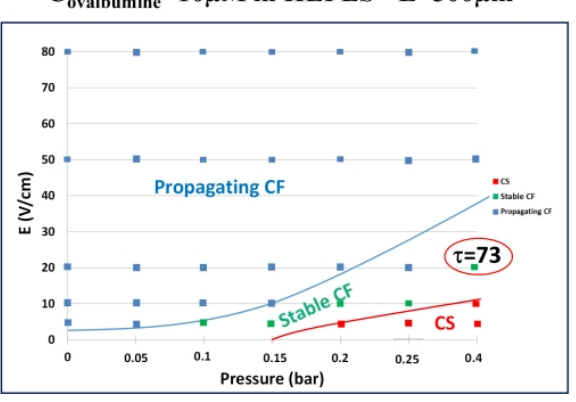

Figure 7. (a) Electropreconcentration profiles of ovalbumin diluted at $10 \mu \mathrm{M}$ in HEPES at $10 \mu \mathrm{M}$ recorded for a nanoslit with $\mathrm{L}=500 \mu \mathrm{m}$ at different coupled $\mathrm{E} / \mathrm{P}$ that produce stable CF regimes, (b) Evolution with time of Cmax the maximum concentration of each profile for $10 \mathrm{~V} / \mathrm{cm}$ and 0.2 bars, and time dependence of its location (distance from the nanoslit), (c) The "Electric field /Pressure" diagram established from ovalbumin electropreconcentration experiments showing at $20 \mathrm{~V} / \mathrm{cm}$ and 0.4 bars the maximal rate $\mathrm{T}=\mathrm{C}_{\max } / \mathrm{C}_{0}=73$ 


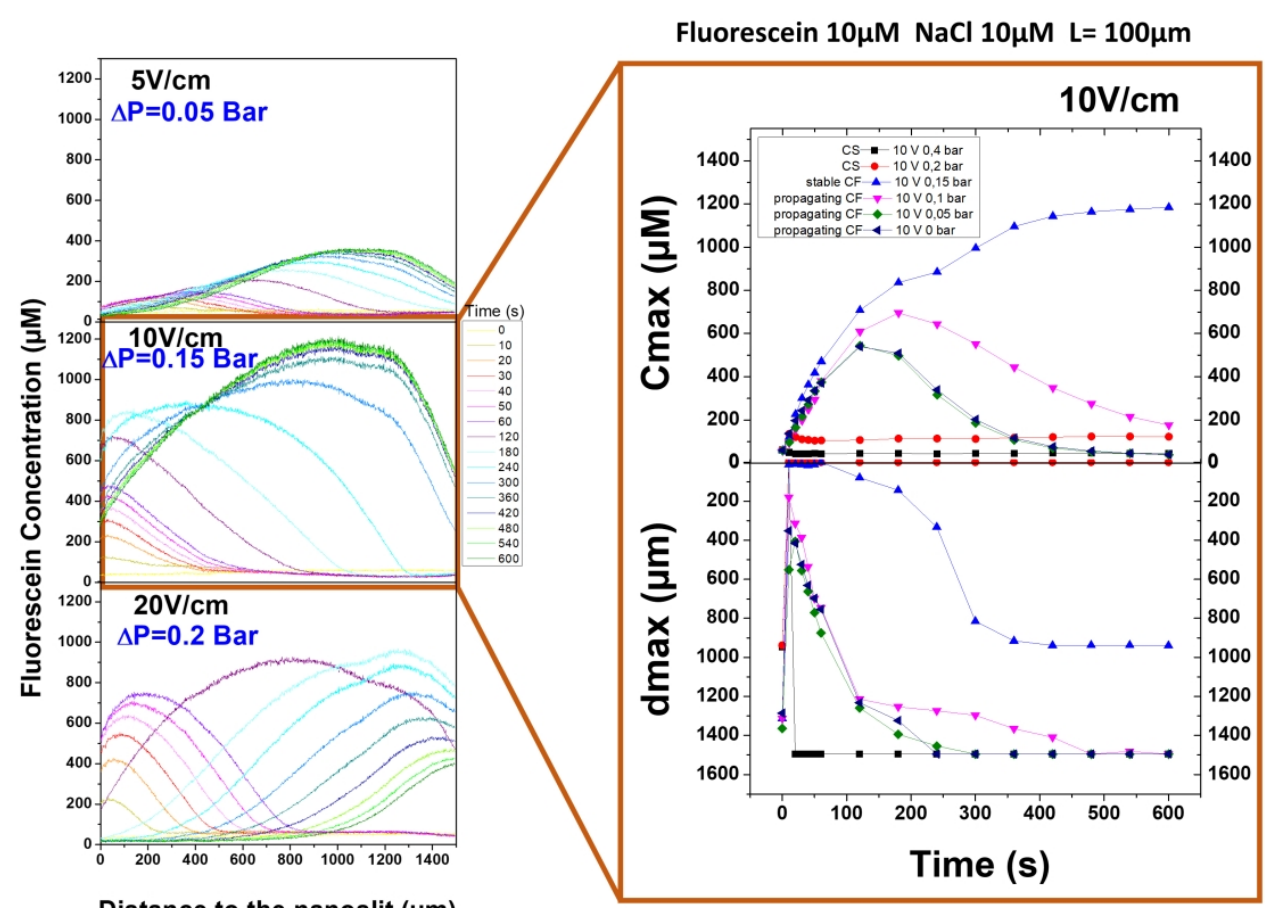

Figure S1.2 - Electropreconcentration profiles recorded at different voltages and different additional hydrodynamic pressures in $\mathrm{NaCl} 10 \mu \mathrm{M}$ with a chip that integrates a $100 \mu \mathrm{m}$ long nanoslit (a) $5 \mathrm{~V} / \mathrm{cm}, 0.05$ bar (b) $10 \mathrm{~V} / \mathrm{cm}, 0.15$ bar and (c) $20 \mathrm{~V} / \mathrm{cm}, 0.2$ bar; (d) at $10 \mathrm{~V} / \mathrm{cm}$, the evolution with time of the maximal concentration $\mathrm{C}_{\max }$ and time dependence of the corresponding distance from the nanoslit $\mathrm{d}_{\max }$. 